\title{
RESULTS OF NORWOOD STAGE I OPERATION: COMPARISON OF HYPOPLASTIC LEFT HEART SYNDROME WITH OTHER MALFORMATIONS
}

Sabine H. Daebritz, MD

Georg D. A. Nollert, MDa

David Zurakowski, $\mathrm{PhD}^{\mathrm{a}}$

Philipe N. Khalil, MD ${ }^{\mathrm{a}}$

Peter Lang, $\mathrm{MD}^{\mathrm{b}}$

Pedro J. del Nido, MD

John E. Mayer, Jr, MD

Richard A. Jonas, MD
Objective: We compared the Norwood stage I operation for hypoplastic left heart syndrome and other complex malformations with ductusdependent systemic circulation. Methods: A retrospective study of 194 patients who underwent a Norwood stage I palliation between 1990 and 1998 was conducted. Malformations in 131 patients were classified as hypoplastic left heart syndrome, defined as aortic and mitral atresia or severe stenosis, normal segmental anatomy, intact ventricular septum, and hypoplasia of the left ventricle. Sixtythree patients had other lesions: hypoplastic left ventricle with ventricular septal defect $(n=18)$, unbalanced complete atrioventricular canal $(n=9)$, complex doubleoutlet right ventricle $(n=14)$, double-inlet left ventricle $(n=11)$, tricuspid atresia with transposition of the great arteries $(n=6)$, and others $(n$ = 5), including heterotaxia. Results: Operative (>30 days) and 1-year survivals were lower for patients with hypoplastic left heart syndrome than for those with other lesions $(63.4 \%$ vs $81 \%, P=.008$, and $51.2 \%$ vs $71.4 \%, P=.02$, respectively). The presence of a nonhypoplastic left ventricle $(\mathrm{n}=27)$ was associated with higher operative and 1-year survivals $(96.3 \%$ vs $64.7 \%, P=.002 ; 88.9 \%$ vs $52.7 \%, P<.001)$. A restrictive atrial septal defect and prematurity tended to increase mortality across both groups. Cox proportional hazards regression indicated that a single right ventricle was the most important independent predictor of death $(P<.001)$. Operative mortality for all patients undergoing the stage I procedure decreased from $38.5 \%(1990-1994)$ to $21.4 \%$ after 1994 ( $P=.02)$. Conclusions: The survival of patients with malformations other than hypoplastic left heart syndrome after the Norwood procedure is greater than for those with hypoplastic left heart syndrome. Staged palliation is valid surgical therapy in these patients, with good results in intermediate follow-up. (J Thorac Cardiovasc Surg 2000; 119:358-67)
$\mathrm{H}$ ypoplastic left heart syndrome (HLHS) is characterized by normal segmental anatomy, aortic and mitral valve stenosis or atresia, and hypoplasia of the left ventricle, ascending aorta, and aortic arch. ${ }^{1}$

From the Departments of Cardiac Surgery ${ }^{\mathrm{a}}$ and Cardiology, Children's Hospital, Boston, Mass.

Read at the Seventy-ninth Annual Meeting of The American Association for Thoracic Surgery, New Orleans, La, April 18-21, 1999.

Received for publication April 22, 1999; revisions requested June 9, 1999; revisions received Oct 5, 1999; accepted for publication Oct $18,1999$.

Address for reprints: Richard A. Jonas, MD, Department of Cardiac Surgery, Children's Hospital, Bader 2, 300 Longwood Ave, Boston, MA 02115 (E-mail: richardjonas@compuserve.com).

Copyright @ 2000 by Mosby, Inc.

$0022-5223 / 2000 \$ 12.00+0 \quad \mathbf{1 2 / 6 / 1 0 3 7 2 9}$
Physiologic features are those of a single right ventricle with unobstructed pulmonary blood flow into welldeveloped pulmonary arteries and a ductus-dependent systemic circulation. Survival is unlikely without surgical therapy. ${ }^{2}$ The pioneering work of Norwood and associates ${ }^{3,4}$ has led gradually to widespread surgical treatment of patients with this malformation. The principles are establishment of a permanent communication between the systemic ventricle and the aorta, relief of systemic outflow obstruction by augmentation of the ascending aorta and arch, restriction of pulmonary blood flow by a modified Blalock-Taussig shunt, and creation of a large interatrial communication.

Various other cardiac malformations share the features of parallel systemic and pulmonary circulations with hypoplasia or obstruction of the systemic outflow, 
Table I. Subgroups of typical HLHS malformations

\begin{tabular}{lrrrcc}
\hline & \multicolumn{2}{c}{ Operative deaths } & & \multicolumn{2}{c}{ Operative survival } \\
\cline { 2 - 3 } Malformation & $n$ & No. & & Percent & $95 \%$ CL \\
\hline AA/MA & 40 & 15 & & 63 & $46-77$ \\
AA/MS & 35 & 12 & & 64 & $48-81$ \\
AS/MS & 53 & 20 & & 62 & $48-75$ \\
Others & 3 & 1 & & 67 & $9-100$ \\
Total & 131 & 48 & & 63 & $55-72$ \\
\hline
\end{tabular}

HLHS, Hypoplastic left heart syndrome; $C L$, confidence limits; $A A$, aortic atresia; $M A$, mitral atresia; $M S$, mitral stenosis; $A S$, aortic stenosis.

unobstructed pulmonary blood flow, and a ductusdependent systemic circulation. In the presence of two well-developed ventricles and atrioventricular valves, surgical treatment aims at a two-ventricle repair. In the absence of one of the two, the Norwood concept of palliation is applicable.

At Children's Hospital, Boston, a variety of malformations other than classic HLHS were treated with the Norwood procedure by creating a pulmonary artery-aortic anastomosis, relieving arch obstruction, and providing pulmonary blood flow by a shunt. The aim of this study was to assess the results of this therapy for this heterogeneous subset of patients. To gain further insights into the pathophysiology, we compared these results with our experience with the Norwood procedure in patients with typical HLHS.

\section{Patients and methods}

The subjects of this retrospective cohort study were all patients who underwent the Norwood stage I palliation between January 1990 and March 1998 at Children's Hospital, Boston $(n=194)$. Patients were excluded in whom a systemic ventricular outflow was created by a pulmonaryaortic anastomosis without aortic arch augmentation. All patients had parallel pulmonary and systemic circulations with ductus-dependent systemic circulation and unobstructed pulmonary blood flow. Cardiac morphology was assessed by review of echocardiographic reports. Typical HLHS was diagnosed in 131 patients who had normal segmental anatomy $\{\mathrm{S}, \mathrm{D}, \mathrm{S}\}$, intact ventricular septum, aortic and mitral atresia or stenosis, and hypoplasia of the left ventricle. The Rhodes criteria ${ }^{5}$ were used to differentiate between patients with HLHS and those with critical aortic stenosis. Table I describes the anatomic subgroups with respect to aortic and mitral valve anatomy. The Norwood procedure was also applied in a second group of 63 patients with other malformations associated with systemic outflow obstruction within the heart and aortic arch obstruction (Table II). Subgroups were hypoplastic left-sided structures with ventricular septal defect (VSD) $(\mathrm{n}=18)$; unbalanced complete atrioventricular canal $(n=9)$; complex double-outlet right ventricle $(n=14)$;
Table II. Pathologic anatomic diagnoses in malformations other than typical HLHS

\begin{tabular}{lccccc}
\hline & \multicolumn{2}{c}{ Operative deaths } & & \multicolumn{2}{c}{ Operative survival } \\
\cline { 2 - 3 } \cline { 5 - 6 } Diagnosis & $n$ & No. & & Percent & $95 \%$ CL \\
\hline $\begin{array}{l}\text { Hypoplastic left heart } \\
\text { structures with VSD }\end{array}$ & 18 & 3 & & 83 & $59-97$ \\
$\begin{array}{l}\text { Unbalanced complete } \\
\quad\end{array}$ & 9 & 3 & & 67 & $30-93$ \\
$\quad$ AV canal & & & & & \\
Complex DORV & 14 & 3 & & 79 & $49-95$ \\
DILV, single LV & 11 & 1 & & 91 & $60-100$ \\
Tricuspid atresia & 6 & 0 & & 100 & $54-100$ \\
$\quad$ with TGA & 5 & 2 & & 60 & $14-95$ \\
$\begin{array}{l}\text { Others (3 heterotaxia) } \\
\text { Total }\end{array}$ & 63 & 12 & & 81 & $69-90$ \\
\hline
\end{tabular}

HLHS, Hypoplastic left heart syndrome; VSD, ventricular septal defect; $A V$, atrioventricular; DORV, double-outlet right ventricle; DILV, doubleinlet left ventricle; $L V$, left ventricle; $T G A$, transposition of the great arteries.

double-inlet left ventricle and single left ventricle $(\mathrm{n}=11)$; tricuspid atresia with transposition of the great arteries $(\mathrm{n}=$ $6)$; and miscellaneous $(n=5)$, including heterotaxia (polysplenia $[\mathrm{n}=2]$ and asplenia $[\mathrm{n}=1])$. A well-developed morphologically left ventricle was present in 27 of these patients; nine of those had two good-sized ventricles ( 1 with complete atrioventricular canal, 4 with complex double-outlet right ventricle, 2 with hypoplastic left-sided structures with VSD, and 2 with miscellaneous malformations). They were not suitable for a two-ventricle repair because of severe stenosis or straddling of one atrioventricular valve.

Mean age and weight at operation were $11 \pm 22$ days (range $0-210$ days, median 5 days) and $3.3 \pm 0.6 \mathrm{~kg}$ (range 2$5.9 \mathrm{~kg}$, median $3.3 \mathrm{~kg}$ ). Age and weight were not significantly different between HLHS and non-HLHS patients (age: median 5 vs 5 days, 25th percentile 3 vs 3 days, 75th percentile 9.5 vs 11.5 days, $P=.70$, Wilcoxon test; weight: 3.35 vs $3.3 \mathrm{~kg}, P=.50)$. Nineteen patients $(10 \%)$ were older than 3 weeks at operation (10 HLHS and 9 non-HLHS patients, $P$ $=0.20), 32.3 \%$ were female, and $67.7 \%$ were male; $29(15 \%)$ were premature with a gestational age of less than 38 weeks (HLHS $10.8 \%$, non-HLHS $23.8 \%, P=0.017$ ). In 67 patients $(34.4 \%)$ the cardiac malformation was diagnosed in utero. Three patients had trisomy 21 and 6 patients had additional extracardiac anomalies (3 HLHS and 3 non-HLHS patients).

Eleven patients $(5.7 \%)$ had a virtually intact atrial septum or a highly restrictive atrial septal defect (ASD), which was enlarged in all but one during cardiac catheterization before surgical palliation. Additional anatomic findings are listed in Table III. One patient had had an attempt at a two-ventricle repair with VSD closure and died after a subsequent conversion to a Norwood procedure. None of the other patients had a previous surgical procedure.

Operative management. The operations were performed by 8 surgeons during the study period.

Surgical technique consisted of the creation of a free com- 
Table III. Preoperative cardiac findings

\begin{tabular}{lccc}
\hline & HLHS & Non-HLHS & P value \\
\hline No. & 131 & 63 & \\
Severe CoA/IAA & $42(32)$ & $44(70)$ & $<.0001$ \\
Restrictive ASD & $8(6)$ & $3(5)$ & .99 \\
Coronary anomalies & $8(6)$ & $5(8)^{\dagger}$ & .76 \\
Coronary fistulas/ & $17(13)$ & $1(2)$ & .008 \\
$\quad$ LV sinusoids & & & \\
Z-score aortic valve* & $-2.2 \pm 2.4$ & $-1.4 \pm 1.2$ & .01 \\
Z-score ascending aorta & $-2.1 \pm 1.1$ & $-1.2 \pm 1.2$ & $<.0001$ \\
Z-score ascending aorta & $-2.3 \pm 2.2$ & $-1.1 \pm 1.6$ & $<.0001$ \\
Z-score transverse arch & $-2.3 \pm 2.4$ & $-2.8 \pm 0.8$ & $<.11$ \\
Z-score mitral valve* & $-3.4 \pm 2.2$ & $-2.0 \pm 2.1$ & $<.0001$ \\
Z-score main pulmonary & $+2.3 \pm 1.7$ & $+2.9 \pm 1.5$ & .02 \\
$\quad$ & & & \\
\hline
\end{tabular}

HLHS, Hypoplastic left heart syndrome; CoA, coarctation of the aorta; IAA, interrupted aortic arch; $A S D$, atrial septal defect; $L V$, left ventricle. Plus-minus values represent the mean and standard deviation. Percentages are shown in parentheses.

"Values do not include Z-scores of atretic valves.

IIncluding 2 patients with anomalous left coronary artery from the pulmonary artery.

munication between the systemic ventricle and the aorta with a pulmonary-aortic anastomosis and arch augmentation with a pulmonary $(41 \%)$ or aortic homograft $(52 \%)$ or other techniques $(7 \%)$. Pulmonary blood flow was provided by a modified Blalock-Taussig shunt of $3 \mathrm{~mm}(4 \%), 3.5 \mathrm{~mm}(85 \%), 4$ $\mathrm{mm}(10 \%)$, or $5 \mathrm{~mm}(1 \%)$. A large interatrial communication was created in all patients. Modifications of the above surgical technique were applied if required by anatomic variants. Cardiopulmonary bypass time was $141 \pm 42$ minutes and circulatory arrest time was $57 \pm 14$ minutes. Secondary sternal closure after the Norwood procedure was frequent.

Postoperative management aimed at achieving balanced systemic and pulmonary blood flow with a pulmonary/systemic flow ratio of $1: 1$ by ventilatory management. Carbon dioxide was not routinely added in the postoperative treatment. Patients were usually extubated 1 or 2 days after sternal closure.

Further surgical therapy included a bidirectional Glenn anastomosis (stage II) before a fenestrated Fontan operation. At the time of follow-up 106 patients had undergone a bidirectional Glenn shunt and 69 had undergone Fontan palliation. Ages were $7.3 \pm 3.3$ months at the stage II palliation and $27.7 \pm 11.2$ months at the third stage and were not significantly different between patients with and without HLHS ( $P$ $=.50$ and $P=.62$, respectively).

Follow-up and statistical analysis. Follow-up was conducted by review of the medical records including postoperative visits at Children's Hospital. If the patient was seen elsewhere, the patient's physicians or guardians were contacted. Patient outcomes included operative, 1-year, and overall survival. Operative mortality was defined as survival of less than 30 days or in-hospital mortality. Follow-up was complete for operative and 1-year survivors except for 2 patients who were less than 1 year of age at the time of the study.
Differences in the proportion of patients with and without preoperative, postoperative, and anatomic variables were compared by $\chi^{2}$ test. Whenever necessary, the Fisher exact test was used. Z-scores were compared by use of the 2-sample Student $t$ test. Approximate 95\% confidence limits for binomial proportions were calculated by the Pratt method. ${ }^{6}$ Actuarial survivals were determined and univariable analysis was performed with the Kaplan-Meier product-limit method. Survival curves were compared by the log-rank test. For multivariable analysis, the Cox proportional-hazards regression model was used to establish the variables independently associated with each outcome. Risk ratios and $95 \%$ confidence intervals were constructed for the significant multivariable predictors. Final models were derived by the backward stepwise likelihood procedure with variables having $P<.20$ in the univariable analyses entered as candidates into the Cox model. Statistical analyses was performed with the SAS software package (version 6.12, SAS Institute, Cary, NC).

The following variables were analyzed: preoperative data (age, weight, fetal diagnosis, prematurity, extracardiac anomalies); anatomic characteristics (subgroups of HLHS and non-HLHS, morphology of the ventricle[s]; Z-scores at different levels of the aorta, of the main pulmonary artery, and of the valves; presence of coarctation of the aorta and interrupted aortic arch, restrictive ASD, coronary anomalies, coronary fistulas, and sinusoids); and operative data (surgeon, different surgical techniques with regard to arch reconstruction and establishment of pulmonary blood flow, and cardiopulmonary bypass and circulatory arrest times).

\section{Results}

Overall survival. Duration of follow-up ranged from 0 to 96.7 months and was $46.2 \pm 3.2$ months for the HLHS patients and $56.3 \pm 4.9$ months for the group with other malformations (log-rank test, $P=.04$ ). Actuarial operative, 1-year, and 3-year survivals in the 194 patients were $69 \% \pm 3 \%, 58 \% \pm 4 \%$, and $54 \% \pm 4 \%$, respectively. Kaplan-Meier survival analysis indicated a significantly shorter mean survival time for HLHS patients than for non-HLHS patients ( $45 \pm 4$ months vs $57 \pm 6$ months, $P=.03$, log-rank test). The other significant predictors included presence of a single right ventricle $(P<$ $.001)$ and prematurity $(P=.01)$. The Cox proportionalhazards model indicated that both prematurity $(P=.02)$ and a single right ventricle $(P<.001)$ were independent predictors of overall mortality.

There were 60 operative deaths. Causes for mortality included myocardial ischemia and unbalanced pulmonary and systemic blood flow resulting in ventricular or systemic organ failure or severe hypoxemia. Twenty-six patients died within the first 24 hours after the operation (20/48 HLHS deaths and 6/12 non-HLHS deaths). Autopsy reports were available in 15 of these patients (10 HLHS; 5 non-HLHS); histologic features 
Table IV. Time and causes of late mortality

\begin{tabular}{|c|c|c|c|c|}
\hline \multirow[b]{2}{*}{ Cause } & \multicolumn{2}{|r|}{$H L H S$} & \multicolumn{2}{|c|}{ Non-HLHS } \\
\hline & No. & Time after stage I (mo) & No. & Time after stage I (mo) \\
\hline \multicolumn{5}{|l|}{ Death before BDG procedure } \\
\hline Sudden, unclear & 7 & $1.5,2.5,4,4,4.5,4.5,5$ & 4 & $1.5,2,2,2.5$ \\
\hline Ventricular failure & 4 & $1.5,2.5,3,{ }^{*} 3.5$ & 1 & 1.5 \\
\hline Cardiac catheterization & 1 & 2 & 0 & \\
\hline Lung disease & 1 & 2.5 & 0 & \\
\hline BDG mortality & 3 & $1.5,,^{\dagger} 2.5,^{\dagger} 5$ & 1 & $2^{\dagger}$ \\
\hline \multicolumn{5}{|l|}{ Death after BDG procedure } \\
\hline Ventricular failure & 3 & $27,42,42^{*}$ & 0 & \\
\hline Cardiac catheterization & 1 & 12 & 0 & \\
\hline Fontan mortality & 0 & & 2 & 34,53 \\
\hline Death after Fontan, unclear & 0 & & 1 & 35 \\
\hline Total & 20 & & 9 & \\
\hline
\end{tabular}

$H L H S$, Hypoplastic left heart syndrome; $B D G$, bidirectional Glenn shunt.

"Status after cardiac transplantation.

'Early bidirectional Glenn shunt at less than 3 months of age.

were compatible with myocardial ischemia in 9 HLHS patients and 1 non-HLHS patient who had an anomalous circumflex coronary artery arising from the pulmonary artery. During the follow-up period, 29 of 134 patients who survived the first-stage palliation died. Causes of late death and the time of their occurrence are described in Table IV. Operative mortality for the bidirectional Glenn procedure was 3.8\% (4/106); three of the operative deaths occurred in patients who were under the age of 3 months at the time of stage II surgery $(39,61$, and 73 days of age). Mortality for the Fontan operation was $2.8 \%(2 / 69)$. There was no significant difference in mortality for patients with or without HLHS for the stage II and Fontan procedures $(P=.99$ and $P=.15$ ).

Operative mortality. Operative mortality was significantly lower for patients with lesions other than typical HLHS (19\% vs 36.6\%, $P=.008$, log-rank test) (Fig 1). Actuarial survival was significantly better in patients with a well-developed morphologically left ventricle in univariate Kaplan-Meier analysis $(P=$ .002) and multivariate Cox regression analysis $(P=$ .01) (Fig 2). Operative survival was $65 \%$ for patients with a single right ventricle, $100 \%$ for patients with two well-developed ventricles, and $95 \%$ for patients with a single morphologically left ventricle $(P=.01)$. The estimated risk for patients with a single right ventricle was approximately four times higher (risk ratio $=3.9$, 95\% confidence intervals $=1.8-9.4)($ Table V).

Prematurity and a virtually intact atrial septum or a highly restrictive ASD were associated with lower operative survival $\left(\chi^{2}\right.$ test: $P=0.02$ and $P=.04$; Kaplan-Meier analysis: $P=.17$ and $P=.1$, respective- ly; Table V). There was no significant difference in survival between the anatomic subgroups of the HLHS patients. This finding also applied to the anatomic subgroups of the non-HLHS patients.

To test the hypothesis that evolving management of patients undergoing the Norwood operation influenced mortality, we divided the patients according to the date of operation into a group operated on during the first 4 years $(1990-1994 ; \mathrm{n}=109 ; 83$ HLHS and 26 nonHLHS patients) and a group operated on during the second 4 years $(1995-1998 ; \mathrm{n}=85 ; 48$ HLHS and 37 non-HLHS patients) of the study period. Operative survival increased from $61.5 \%$ in the first period to $78.6 \%$ for patients in the latter period $(P=.01$, univariate Kaplan-Meier analysis; $P=.02$, multivariate Cox regression analysis) (Fig 3). Survival improved in the second study period for HLHS patients $(P=.04)$ and did not change for the non-HLHS patients $(P=.75)$.

No other variable correlated with operative survival. Analysis included preoperative data, anatomic characteristics, and operative data.

One-year survival. One-year survival was significantly higher for malformations other than typical HLHS ( $71 \%$ vs $51 \%, \chi^{2}$ analysis, $P=.03$; KaplanMeier, $P=.02$ ). Once again, the presence of a welldeveloped morphologically left ventricle was a predictor for survival in univariate $(P<.001)$ and multivariate analysis $(P=.008)$. Patients with a single right ventricle were estimated to have a risk nearly 5 -fold higher for 1 -year mortality (risk ratio $=4.8,95 \%$ confidence intervals $=2.0-12.7)($ Table V). Prematurity and a restrictive ASD at birth impaired survival $(P=.04$ and $P=.02$, respectively, in $\chi^{2}$ analysis; $P=.02$ and $P=$ 


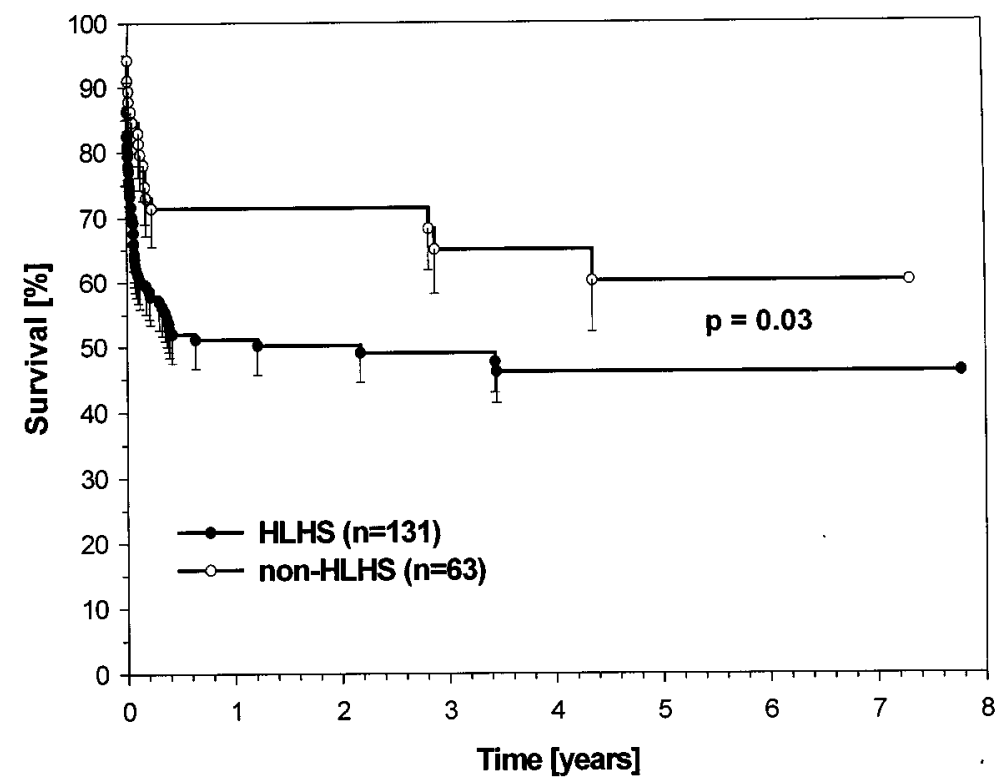

$\begin{array}{lrrrrrrr}\text { number of patients: } & & & & & \\ \text { HLHS } & 67 & 46 & 35 & 27 & 20 & 14 & 6 \\ \text { non-HLHS } & 44 & 27 & 18 & 14 & 9 & 4 & 1\end{array}$

Fig 1. Survival after stage I procedure for HLHS and non-HLHS patients. Each step in the curve denotes an event, and at each event the standard error is indicated by vertical bars. The curves show a sharp decline in survival until the age of 6 months, at which time the graphs flatten out.

.20 , respectively, in Kaplan-Meier analysis). None of the other variables, including date of operation, correlated with 1-year survival.

During follow-up, 5 patients underwent cardiac transplantation with 2 deaths (Table IV). The 3 survivors had undergone transplantation after the second step (2 patients) and after the Fontan procedure (1 patient); all patients undergoing transplantation were in the HLHS group.

\section{Discussion}

HLHS is a lethal anomaly if not treated surgically. The Norwood operation is predominantly an extracardiac procedure that can be applied to a variety of malformations regardless of intracardiac anomalies. The results have improved continuously because of increasing surgical experience and better understanding of the delicate balancing of pulmonary and systemic blood flow in the perioperative period, as well as improvements in techniques of cardiopulmonary bypass in neonates. Operative survival was $69 \%$ in our study over a period of 8 years with significant improvement to $79 \%$ after 1994. Further improvement has occurred beyond the end point of this study. From January to December 1998, only 1 of 21 patients died after stage I palliation. A learning curve is expected in the treatment of these challenging patients and is attributed to increasing experience both in surgical and in perioperative management. Therefore, concentration of patients in larger volume centers would seem to be advantageous. $^{7}$

Early mortality is often due to myocardial ischemia or imbalance of pulmonary and systemic blood flow with subsequent organ failure or hypoxemia. ${ }^{8}$ Mortality after initial hospital discharge is not insignificant and reflects the delicately balanced nature of the circulation in patients after the first step of staged palliation.

Several studies have been performed to identify predictors of outcome of the Norwood procedure. Cardiopulmonary bypass time, ${ }^{9}$ prerepair $\mathrm{pH}$, lower birth weight and associated noncardiac anomalies, ${ }^{10}$ severe obstruction to pulmonary venous return, ${ }^{11}$ diminutive ascending aorta, ${ }^{12}$ and anatomic subtype, specifically aortic and mitral atresia, ${ }^{13}$ have been demonstrated to impair survival in recent studies. None of these risk factors, however, has been consistent 


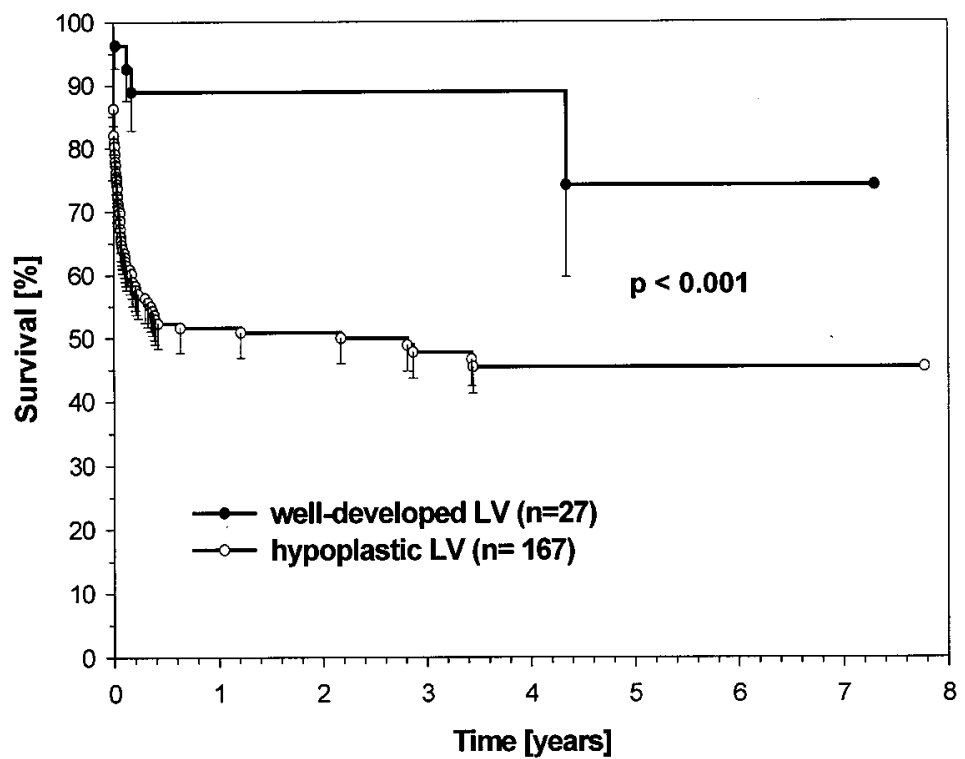

$\begin{array}{lccccccc}\text { number of patients } & & & & & & 1 \\ \text { well-developed LV } & 24 & 16 & 10 & 6 & 4 & 2 & 6 \\ \text { hypoplastic LV } & 87 & 59 & 43 & 35 & 25 & 16 & 6\end{array}$

Fig 2. Survival after stage I procedure for patients with a left ventricle and without a left ventricle. The presence of a morphologically left ventricle was the most important predictor of operative and 1-year survival. $L V$, Left ventricle.

Table V. Predictors of outcome

\begin{tabular}{|c|c|c|c|c|c|}
\hline \multirow[b]{2}{*}{ Variable } & \multicolumn{2}{|c|}{ Univariable Kaplan-Meier analysis } & \multicolumn{3}{|c|}{ Multivariable Cox proportional hazards analysis } \\
\hline & Association & $\mathrm{P}$ value & Risk ratio & $95 \% C I$ & $\mathrm{P}$ value \\
\hline \multicolumn{6}{|l|}{ Operative mortality } \\
\hline HLHS & + & .008 & & & .34 \\
\hline Single RV & + & .002 & 6.0 & $2.3-27.8$ & .01 \\
\hline Restrictive ASD & + & .10 & & & .16 \\
\hline Prematurity & + & .17 & & & .13 \\
\hline DOS (1990-1994) & + & .01 & 2.2 & $1.3-4.4$ & .02 \\
\hline \multicolumn{6}{|l|}{ One-year mortality } \\
\hline HLHS & + & .02 & & & .48 \\
\hline Single RV & + & $<.001$ & 4.8 & $2.0-12.7$ & .008 \\
\hline Restrictive ASD & + & .20 & & & .29 \\
\hline Prematurity & + & .02 & 2.3 & $1.5-4.0$ & .01 \\
\hline DOS (1990-1994) & + & .08 & & & .23 \\
\hline \multicolumn{6}{|l|}{ Overall mortality } \\
\hline HLHS & + & .03 & & & .65 \\
\hline Single RV & + & $<.001$ & 3.9 & $1.8-9.4$ & $<.001$ \\
\hline Restrictive ASD & + & .15 & & & .14 \\
\hline Prematurity & + & .01 & 2.1 & $1.3-3.8$ & .02 \\
\hline DOS (1990-1994) & + & .07 & & & .22 \\
\hline
\end{tabular}

$C I$, Confidence interval; $H L H S$, hypoplastic left heart syndrome; $R V$, right ventricle; $A S D$, atrial septal defect; $D O S$, date of surgery.

across all studies. In contrast to our previous reports, ${ }^{13}$ the size of the aortic root and ascending aorta was not a predictor of survival in this series. However, mortali- ty in the first 24 hours after the operation related to myocardial ischemia seemed to be more frequent in HLHS patients. 


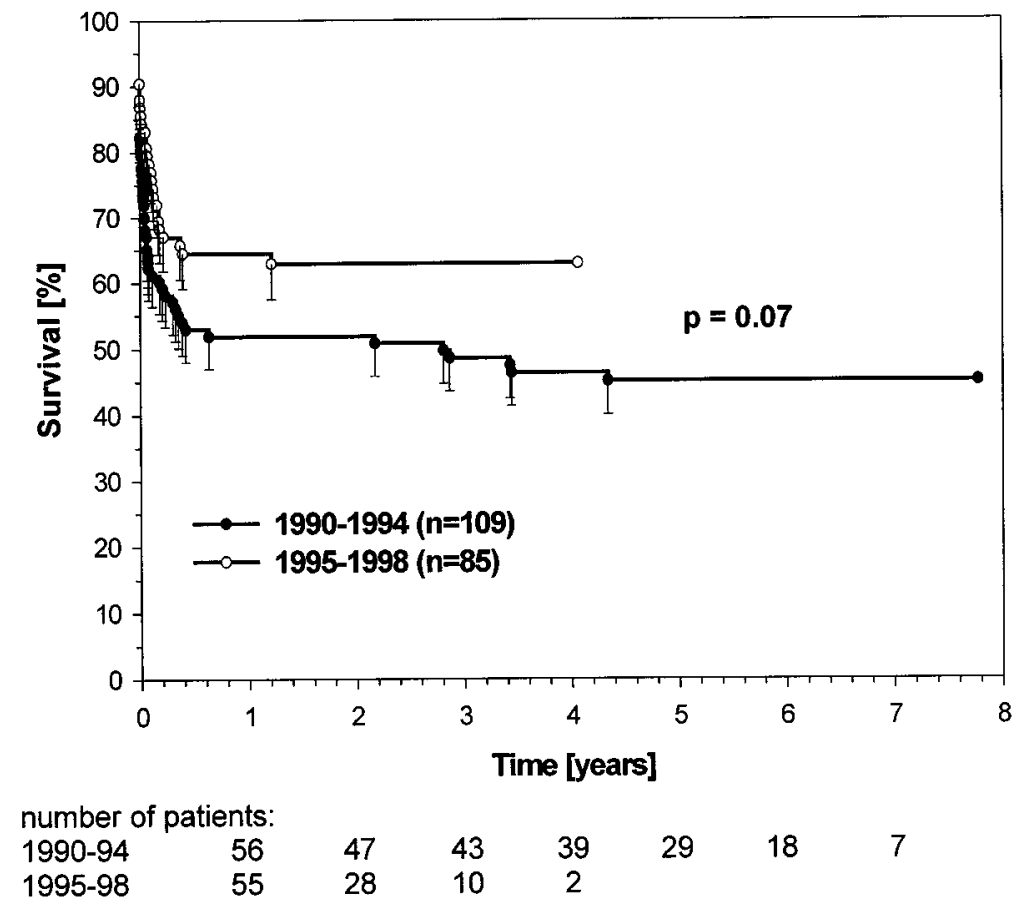

Fig 3. Survival after stage I procedure for patients operated on before 1995 and for patients operated on from 1995 to 1998. Increasing experience both in surgical and perioperative management led to the observed improvement in survival.

In our study, prematurity and a restrictive ASD tended to influence operative mortality. With HLHS physiology, restrictive flow across the atrial septum causes pulmonary venous hypertension. Although catheterdirected atrial septoplasty is usually performed shortly after admission, pulmonary vascular resistance may be increased, making the balance between systemic and pulmonary blood flow more difficult to achieve in the early postoperative period. ${ }^{14,15}$

We compared the outcome of typical HLHS with that of other malformations with the same pathophysiology and found a greater operative and 1-year survival in the non-HLHS patients. Actuarial survival in HLHS patients was in the same range as that in patients with aortic atresia after staged reconstructive surgery as reported by Jacobs, Blackstone, and Bailey ${ }^{10}$ in the multicenter study of the Congenital Heart Surgeons Society $(64 \%, 50 \%$, and $47 \%$ after 1 , 12 , and 36 months). In that report, survival was higher for patients who entered the transplant protocol ( $85 \%, 64 \%$, and $62 \%$ after 1,12 , and 36 months) and is comparable with that of our non-HLHS patient group.

In our study the presence of a morphologically left ventricle was the most important predictor of survival in this time frame, although this has not been a consistent finding in our previous reports.

In the neonatal period and during the first weeks after birth, the myocardium has the potential for hyperplasia, which represents real growth of muscle cells. ${ }^{16}$ This ability may be different between the right and left ventricles, adding to the improved outcome of stage I palliation in newborn infants with anatomically left ventricles. A tricuspid atrioventricular valve with possibly associated abnormalities may also contribute to the limited prognosis of patients with a single right ventricle. ${ }^{17,18}$ In the presence of two ventricles with antegrade flow through the aortic valve or a VSD, the left ventricle can contribute to cardiac output, thus decreasing the workload of the right ventricle. Different contraction patterns of a right ventricle during the cardiac cycle in the absence of a second ventricle have recently been described and may also influence outcome. ${ }^{19}$

In contrast to our findings, Jacobs and coworkers ${ }^{20}$ described a comparable outcome for non-HLHS and HLHS patients. However, they used a different classification of these complex malformations and did not distinguish between patients with right, left, or two ventricles. Kanter and colleagues ${ }^{21}$ also applied the Norwood concept successfully to patients with variants 
of the HLHS. Mosca and associates ${ }^{22}$ reported an $89 \%$ operative survival for the Norwood procedure in tricuspid atresia or double-inlet right ventricle and transposition of the great arteries with arch anomalies. These patients represent two subsets of our non-HLHS patient group with well-developed left ventricles, confirming its impact on survival. Comparison of the subgroups of the non-HLHS patients in a larger series would help to further determine predictors of survival, which might be eliminated in our analysis by the heterogeneity of malformations.

Some anatomic subsets of the HLHS and non-HLHS patients have been considered potential candidates for a biventricular repair. Tchervenkov and associates ${ }^{23}$ selected patients with severe hypoplasia of the leftsided structures but with antegrade flow through the left side of the heart and performed a biventricular repair. Steger and coworkers ${ }^{24}$ combined Norwood and Rastelli procedures for interrupted aortic arch with subaortic stenosis. The criteria to opt for a Norwood procedure in complex malformations are not well defined. Systematic analysis of mortality for a two-ventricle approach versus a one-ventricle approach is so far only available for critical aortic stenosis. We consider the possibility of a biventricular repair whenever feasible. However, the mortality for single ventricle palliation after a failed attempt at a two-ventricle repair has been exceedingly high in our experience, possibly because of elevation of pulmonary venous pressure. Thus the decision for a two-ventricle versus a one-ventricle approach must be made accurately early in the neonatal period.

Biventricular repair after a stage I operation and a salvage Norwood procedure to bridge a period of severe left ventricular dysfunction has been discussed. ${ }^{19}$ The potential of a hypoplastic left ventricle to grow and the conditions under which this might happen are intriguing. We have so far not observed remarkable growth of a hypoplastic left ventricle in any of our patients after stage I palliation. This may be due at least in part to the presence of an unrestricted interatrial communication and the worse compliance of the hypoplastic left ventricle.

In summary, the findings of our study confirm the validity of the Norwood concept in the treatment of complex cardiac anomalies with good results at intermediate follow-up.

\section{REFERENCES}

1. Noonan JA, Nadas AS. The hypoplastic left heart syndrome: an analysis of 101 cases. Pediatr Clin North Am 1958;5:1029-56.

2. Castaneda AR, Jonas RA, Mayer JE, Hanley FL. Hypoplastic left heart syndrome. In: Castaneda AR, Jonas RA, Mayer JE, Hanley FL, editors. Cardiac surgery of the neonate and infant. Philadelphia: WB Saunders; 1994. p. 363-86.

3. Norwood WI, Kirklin JK, Sanders S. Hypoplastic left heart syndrome: experience with palliative surgery. Am J Cardiol 1980; 45:87-91.

4. Norwood WI, Lang P, Hansen D. Physiologic repair of aortic atresia-hypoplastic left heart syndrome. N Engl J Med 1983; 308:23-6.

5. Rhodes LA, Colan SD, Perry SB, Jonas RA, Sanders SP. Predictor of survival in neonates with critical aortic stenosis. Circulation 1991;84:2325-35.

6. Blyth CR. Approximate binomial confidence limits. J Am Stat Assoc 1986;81:843-55.

7. de Leval MR, Francois K, Bull C, Brawn W, Spiegelhalter D. Analysis of a cluster of surgical failures: application to a series of a neonatal arterial switch operations. J Thorac Cardiovasc Surg 1994;107:914-23.

8. Bartram U, Grünefelder J, Van Praagh R. Causes of death after the modified Norwood procedure: a study of 122 postmortem cases. Ann Thorac Surg 1997;64:1795-822.

9. Kern JH, Hayes CJ, Michler RE, Gersony WM, Quaegebeur JM. Survival and risk factor analysis for the Norwood procedure for hypoplastic left heart syndrome. Am J Cardiol 1997;80:170-4.

10. Jacobs ML, Blackstone EH, Bailey LL. Intermediate survival in neonates with aortic atresia: a multi-institutional study. J Thorac Cardiovasc Surg 1998;116:417-31.

11. Bove EL, Lloyd TR. Staged reconstruction for hypoplastic left heart syndrome: contemporary results. Ann Surg 1996;224:38794; discussion 394-5.

12. Bando K, Turrentine MW, Sun Kyung, Sharp TG, Caldwell RL, Darragh RK, et al. Surgical management of hypoplastic left heart syndrome. Ann Thorac Surg 1996;62:70-7.

13. Jonas RA, Hansen DD, Cook N, Wessel D. Anatomic subtype and survival after reconstructive operation for hypoplastic left heart syndrome. J Thorac Cardiovasc Surg 1994;107:1121-8.

14. Atz AM, Feinstein JA, Jonas RA, Perry SB, Wessel DL. Preoperative management of pulmonary venous hypertension in hypoplastic left heart syndrome with restrictive atrial septal defect. Am J Cardiol 1999;83:1224-8.

15. Canter CE, Moorehead S, Huddleston CB, Spray TL. Restrictive atrial septal communication as a determinant of outcome of cardiac transplantation for hypoplastic left heart syndrome. Circulation 1993;88(5 Pt 2):II456-60.

16. Bujaisky L, Zak R. Biological mechanisms of hypertrophy. In: Fozzard HA, Haber E, Jennings RB, et al, editors. The heart and cardiovascular system. New York: Raven Press; 1986. p. 1491.

17. Gentles TL, Mayer JE Jr, Gauvreau K, Newburger JW, Lock JE, Kupferschmidt JP, et al. Fontan operation in five hundred consecutive patients: factors influencing early and late outcome. J Thorac Cardiovasc Surg 1997;114:376-91.

18. Stamm C, Anderson RH, Ho SY. The morphologically tricuspid valve in hypoplastic left heart syndrome. Eur J Cardiothorac Surg 1997;12:587-92.

19. Fogel MA, Weinberg PM, Fellows KE, Hoffmann EA. A study in ventricular-ventricular interaction: single right ventricles compared with systemic right ventricles in a dual-chamber circulation. Circulation 1995;92:219-30.

20. Jacobs ML, Rychnik J, Murphy JD, Nicolson SC, Steven JM, Norwood WI. Results of Norwood's operation for lesions other 
than hypoplastic left heart syndrome. J Thorac Cardiovasc Surg 1995;110:1555-62.

21. Kanter KR, Miller BE, Cuadrado AG, Vincent RN. Successful application of the Norwood procedure for infants without hypoplastic left heart syndrome. Ann Thorac Surg 1995;59:3014.

22. Mosca RS, Hani AH, Kulik TJ, Crowley DC, Michelfelder EC, Ludomirsky A, et al. Modified Norwood operation for single left ventricle and ventriculoarterial discordance: an improved surgical technique. Ann Thorac Surg 1997;64:1126-32.

23. Tchervenkov CI, Tahta SA, Jutras LC, Beland MJ. Biventricular repair in neonates with hypoplastic left heart complex. Ann Thorac Surg 1998;66:1350-7.

24. Steger V, Heinemann MK, Brenndorff CI, Ziemer G. Combined Norwood and Rastelli procedure for repair of interrupted aortic arch with subaortic stenosis. Thorac Cardiovasc Surg 1998;46: 156-8.

\section{Discussion}

Dr William I. Norwood (Wilmington, Del). The group from the Children's Hospital of Boston has analyzed a cohort of patients with HLHS and a smaller but very substantial group of patients with a variety of non-HLHS anatomic abnormalities, all of whom underwent a similar palliative operation over an 8-year period.

Over the years, I have found it more awkward to deal surgically with aortopulmonary relations such as aortic atresia with transposition of the great arteries and tricuspid atresia. However, as we evaluated these different anatomic variations in comparison to HLHS treated with a similar palliative operation, we never really noticed a consistent difference between outcomes of those two patient groups.

It is always difficult to discuss a very diverse set of patients, and it is difficult to be sure that one is making meaningful inferences even when our statistical packages encourage us to infer. The principal conclusion that I hear, at least on this group of patients, is that non-HLHS patients fare better than HLHS patients; having some semblance of a left ventricle is advantageous.

My difficulty with such information is that I do not know what to do with it. Do we use it to select patients? Do we strongly consider transplantation when we are dealing with a dominant right ventricle? Or do we simply try harder?

I made a slide comparing some of our data with your data, to address this particular point, but as I read the first paragraph in the discussion of this manuscript, I found the point is made. You state: "HLHS is a lethal anomaly if not treated surgically. The Norwood operation is predominantly an extracardiac procedure that can be applied to a variety of malformations regardless of intracardiac anomalies. The results have improved continuously because of increasing surgical experience and better understanding of the delicate balancing of pulmonary and systemic blood flow in the perioperative period, as well as improvements in techniques of cardiopulmonary bypass in neonates. Operative survival was $69 \%$ in our study over a period of 8 years with significant improvement to $79 \%$ after 1994. Further improvement has occurred beyond the end point of this study. From January to December 1998, only 1 of 21 patients died after stage I palliation." I do not think the right ventricle is getting stronger. I think you are on the right track. You further state: "A learning curve is expected in the treatment of these challenging patients and is attributed to increasing experience both in surgical and in perioperative management."

Two interesting points came out that I would like to discuss. I am not sure you can come up with a concrete answer to these two questions. I noticed that a group of 11 patients had virtually intact atrial septum or very restrictive atrial septum, and they had cardiac catheterization and balloon manipulation of the atrial septum preoperatively. We have given up trying to deal with the virtually intact atrial septum in the catheterization laboratory because of its relative thickness and the relative smallness of the left atrium. We actually have altered the timing of the operation rather than going to the cardiac catheterization laboratory before surgical intervention. In your 11 patients, do you think it was worthwhile? Did it favorably influence the outcome and the physiology of the patients after the operation?

Second, a number of patients died in the interim between the initial palliation and bidirectional Glenn procedure. We continue to use predominantly a 4-mm tube graft or shunt, and I noticed that you used predominantly a $3.5-\mathrm{mm}$ shunt. What are your thoughts relating to the interim mortality and the size of the shunt? How would that influence the interim mortality? Would it lead patients to an earlier bidirectional Glenn shunt or obviate interim myocardial dysfunction, which is probably even worse than increasing cyanosis?

Dr Daebritz (Boston, Mass). Thank you for your comments. It is interesting that the risk factors for stage I mortality are inconsistent between different studies. A variable that reaches significance in one series does not show an influence in another series. It may be that multiple factors play a role in the stage I mortality or that we are missing a major confounding variable.

We found a difference in survival of our Norwood patients with regard to the underlying anatomy; results of other studies do not confirm this. This might be due to different patient selection, as it is controversial how to define HLHS and where to draw the line to non-HLHS anatomy. Additionally, the group of non-HLHS patients in itself is inhomogeneous, with only small numbers for each anatomic subgroup. Therefore, differences in survival between the subgroups might not be detected by statistical analysis or might be eliminated when comparing the whole group of non-HLHS patients to HLHS patients. Nevertheless, in our series patients with a well-developed left ventricle had a better survival.

What do we do with this information? We do not think we can use it for selecting patients for staged palliation or transplantation. We do think, however, that these results are of importance in borderline patients, in whom a two-ventricle repair is considered an option. We found a good survival for the Norwood palliation in these patients. Attempts at a twoventricle repair have to compete with these results. A much 
higher mortality would not be acceptable, although some patients might end up with two-ventricle physiology.

A highly restrictive ASD or a virtually intact atrial septum causes low cardiac output with severe acidosis and pulmonary venous hypertension. We prefer stabilizing the patient before the operation instead of operating with the patient in poor clinical condition. Blade septostomy has been performed but carries a high risk of damaging the free atrial wall. Usually a balloon atrial septoplasty through a separate hole in the atrial septum is successful. In most cases a stable preoperative clinical situation without low output and acidosis can be accomplished with this strategy.

Regarding the shunt sizes, the current policy at Children's Hospital in Boston is to use 3.5-mm shunts. We think that this creates the best balance between pulmonary and systemic circulation, at least in the early postoperative period. This might change when the patient grows. The interim mortality between stage I and stage II is probably due in part to disturbance of the delicate balance between the two circulations. The bidirectional Glenn shunt leads to a stable relation of pulmonary and systemic blood flow without shunt dependence of pulmonary perfusion. Additionally, volume unloading of the ventricle and higher diastolic systemic pressures with improved coronary perfusion help to preserve myocardial function. After this step, mortality decreases sharply. Therefore, the interim mortality after the stage I operation is related to obstructed flow through the shunt, ventricular dysfunction, or myocardial ischemia. We do not have detailed information about the causes of death in most of the cases, as the patients often die suddenly outside the hospital and autopsies are not available. To find out whether or not shunt size plays a role, we would need a detailed analysis of each patient dying in that time period. However, if shunt size was the major cause of this interim mortality, one would expect to find series with no significant mortality between the first two stages. These do not yet exist. Probably the balanced perfusion of body and lung under Norwood stage I physiology is so delicate that it can be disturbed by common conditions like respiratory and pulmonary infections or fever.

It might be reasonable to try to decrease this mortality by performing the bidirectional Glenn shunt earlier after the stage I procedure, for example, with an interval of 3 months. The 3 patients in our series who had a Glenn shunt at an age of less than 3 months and who died did not have a shuntdependent pulmonary perfusion for 3 months. In these patients, severe imbalance of pulmonary and systemic blood flow or myocardial dysfunction forced the surgeon to perform the Glenn shunt very early after the stage I procedure. Performing an elective Glenn operation 3 months after stage I with the patient in good clinical condition might improve the results.

\section{Targeted}

The Journal of Thoracic and Cardiovascular Surgery gives you two tables of contents.

The condensed table of contents tells you at a glance what topics and authors are presented each month. The expanded table of contents gives you a brief abstract of each article. You select only those articles of most interest to you for further reading. 